\title{
Probing Heavy-Light Neutrino Mixing in Left-Right Seesaw Models at the LHC
}

\author{
Chien-Yi Chen, ${ }^{1}$ P. S. Bhupal Dev, ${ }^{2}$ and R. N. Mohapatra ${ }^{3}$ \\ ${ }^{1}$ Department of Physics, Brookhaven National Laboratory, Upton, New York 11973, USA \\ ${ }^{2}$ Consortium for Fundamental Physics, School of Physics and Astronomy, \\ University of Manchester, Manchester M13 9PL, United Kingdom \\ ${ }^{3}$ Maryland Center for Fundamental Physics and Department of Physics, \\ University of Maryland, College Park, Maryland 20742, USA
}

\begin{abstract}
We show that in TeV-scale left-right (L-R) symmetric seesaw models, there are new dominant contributions to the collider signals of heavy Majorana neutrinos arising from the heavy-light neutrino mixing, which directly probe the seesaw matrix in a certain class of models. We propose a way to distinguish this contribution from the widely discussed one that only probes the Majorana nature of the heavy right-handed neutrinos, by analyzing some simple kinematical variables. We find that in this class of L-R seesaw models the existing LHC data already yield slightly stronger constraints on the heavy-light neutrino mixing than those derived for standard seesaw models, and the improvement will be significant as more data are collected.
\end{abstract}

\section{INTRODUCTION}

The neutrino oscillation data unambiguously establish that neutrinos have tiny but non-zero masses, the explanation of which calls for physics beyond the Standard Model (SM). A simple paradigm for understanding the smallness of left-handed (LH) neutrino masses is the (type-I) seesaw mechanism [1] where one introduces a set of heavy SM singlet Majorana fermions $N$ breaking the $(B-L)$-symmetry. The seesaw matrix has the generic form in the $\left(\nu_{L}, N\right)$ space:

$$
\left(\begin{array}{cc}
0 & m_{D} \\
m_{D}^{\top} & M_{N}
\end{array}\right)
$$

where $m_{D}$ is the Dirac mass term which mixes the $\nu$ and $N$ states, and $M_{N}$ is the Majorana mass term for $N$. This leads to the seesaw formula for light neutrinos of the form [1]

$$
M_{\nu} \simeq-m_{D} M_{N}^{-1} m_{D}^{\top},
$$

and a heavy-light neutrino mixing of order $m_{D} M_{N}^{-1}$ [2]. Thus there are two key aspects to the seesaw mechanism: the Majorana mass of the heavy neutrino, and the mixing between the heavy and light neutrinos. To probe the seesaw paradigm experimentally, one must therefore test both the Majorana nature of $N$ and the heavy-light neutrino mixing effects. There are two possible ways to do this. The first well known way is to test for the Majorana nature of both the heavy and light neutrino masses via searches for the neutrinoless double beta decay $(0 \nu \beta \beta)$ and disentangle the heavy neutrino effect [3] which however does not necessarily probe the heavy-light neutrino mixing. The second way is to directly look for the presence of heavy-light mixing, which can manifest in several ways, e.g., (i) via departures from unitarity of the PMNS neutrino mixing matrix 4, which can be probed in neutrino oscillation experiments as well as lepton flavor violation (LFV) searches, and (ii) via their signatures in colliders [5]. Clearly for these latter tests of seesaw to be effective, the mixing parameter $m_{D} M_{N}^{-1} \equiv V_{\ell N}$ must be significant and this requires that $M_{N}$ must be small (in the $\mathrm{TeV}$ range) and $m_{D}$ large (in the few $\mathrm{GeV}$ range) simultaneously. It is the second aspect of testing seesaw at colliders that we focus on in this paper.

To proceed with details, we remind the reader that the simplest implementation of the seesaw paradigm is to add the gauge-singlet neutrino field $N$ with a Majorana mass $M_{N}$ to the SM. The seesaw scale (synonymous with $M_{N}$ ) then remains an adhoc parameter unconnected to any new physics or symmetry. We will call this scenario the SM-seesaw in what follows. On the other hand, these heavy neutrinos $N$ naturally arise as the right-handed $(\mathrm{RH})$ partners of the LH neutrinos in the Left-Right (L-R) symmetric extension of the SM which was originally introduced [6] in order to understand the origin of parity violation in weak interactions at low energies. The minimal L-R symmetric theory, based on the $S U(3)_{c} \times S U(2)_{L} \times S U(2)_{R} \times U(1)_{B-L}$ gauge group, provides a natural explanation of the seesaw scale as connected to the $S U(2)_{R} \times U(1)_{B-L}$ breaking scale. The smallness of the LH neutrino mass in these theories is connected to the extent to which the RH-current effects in weak interactions are suppressed at low energy. We will call this scenario the L-R seesaw. Thus, a TeV-scale L-R symmetric theory provides an attractive class of seesaw models that can be probed at the LHC [7].

As noted, for the case of SM-seesaw, the Majorana mass $M_{N}$ is hard to test in colliders without the help of the heavy-light neutrino mixing $V_{\ell N}$. The full seesaw mechanism can then manifest itself as final states with same-sign dileptons plus two jets without missing energy $\left(\ell^{ \pm} \ell^{ \pm} j j\right)$, arising from the Feynman diagram shown in Fig. 17. This signal depends crucially on the heavylight mixing and can effectively probe the heavy neutrino masses $M_{N}$ only up to a few hundred $\mathrm{GeV}$ as has been extensively discussed in the literature 8. It must be stressed that any positive signal would not only signify the Majorana character of the heavy sub-TeV neutrino $N$ but also a specific non-generic structure of $m_{D}$. The reason is that in generic ("vanilla") seesaw case, we expect the heavy-light mixing $V_{\ell N} \sim \sqrt{m_{\nu} / M_{N}}$ which is very 


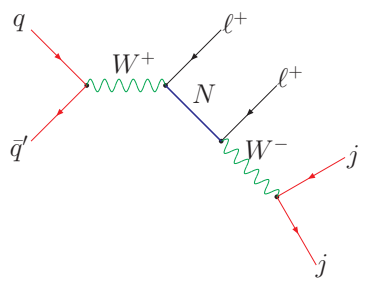

(a) $L L$

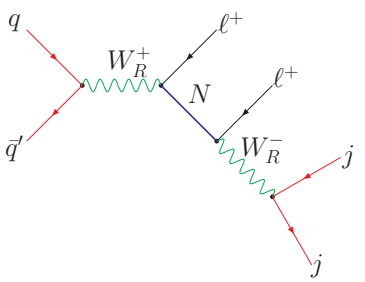

(b) $R R$

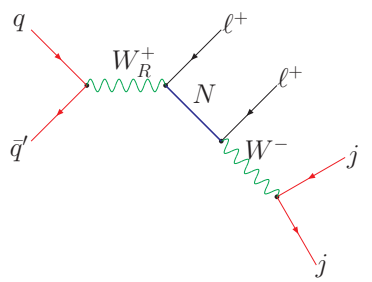

(c) $R L$

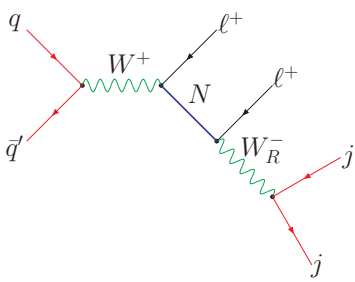

(d) $L R$

FIG. 1. The Feynman diagrams contributing to the 'smoking gun' collider signal of seesaw in the minimal L-R model.

tiny for TeV-scale $M_{N}$ due to the smallness of the light neutrino masses (the current upper limit on $m_{\nu} \leq 0.1$ $\mathrm{eV}$ [9]), making the collider signal unobservable. Only if the Dirac matrix $m_{D}$ in Eq. (2) has specific forms (see e.g., [10, 11]) can $V_{\ell N}$ be significant enough to have observable lepton number violation (LNV) at the LHC 8 ]. The latter can reveal underlying symmetries of the lepton sector, which will be an important step towards a full understanding of the neutrino mass physics. We note parenthetically that the other manifestation of LNV, namely $0 \nu \beta \beta$, receives dominant contribution only from the light neutrino mass in this case 12] (except when the light neutrino contribution vanishes due to cancellation [11, 13]).

However, in the L-R symmetric embedding of $\mathrm{TeV}$ scale seesaw, the presence of $\mathrm{RH}$ gauge interactions lend considerable richness to the manifestations of seesaw in experiments [14. Not only are there new contributions to $0 \nu \beta \beta$ from $\mathrm{RH}$ gauge bosons $\left(W_{R}\right)$ [15], but the profile of seesaw manifestation at colliders changes dramatically [16]. In fact, the $\ell^{ \pm} \ell^{ \pm} j j$ signal now receives three new contributions from different combinations of $W_{R}$ exchange and heavy-light neutrino mixing (Figs. 1 $\mathrm{b}-1 \mathrm{~d}$ ). The contribution which arises from the exchange of two $W_{R}$ bosons is the one that has been widely discussed for the L-R seesaw case [17]. However for certain specific textures of Dirac mass matrix, which lead to an enhanced heavy-light neutrino mixing, the profile of the "smoking gun" $\ell^{ \pm} \ell^{ \pm} j j$ signal changes drastically. The goal of this paper is to explore the relative magnitude of the heavy-light mixing contribution compared to the $W_{R} W_{R}$ contribution at the LHC and assess their impact on our understanding of the seesaw paradigm.

Important for the collider discussion are the relative values of the $W_{R}$ and $N$ masses. There are theoretical arguments based on vacuum stability [18] which suggest that the heavy neutrinos in the minimal L-R seesaw model are lighter than the RH gauge bosons for a large range of parameters. We will therefore consider this mass ordering $M_{N}<M_{W_{R}}$ in this paper (although going beyond the minimal version, one could avoid this restriction). A major implication of this, as shown in this paper, is that for $\mathrm{RH}$ gauge boson masses below $4-5 \mathrm{TeV}$, when it can be produced at the $\sqrt{s}=14 \mathrm{TeV}$ LHC with a decent cross section, its decay to the on-shell heavy $\mathrm{RH}$ neutrinos will allow a new probe of its mixing with the light neutrinos for a wider heavy neutrino mass range of up to a few TeVs from a study of $\ell^{ \pm} \ell^{ \pm} j j$ final states. This information, together with the light neutrino mixing parameters extracted from neutrino oscillation data, should suffice to fully determine the Dirac mass matrix in the minimal L-R model, and hence, facilitate its testability in other low energy experiments.

\section{TEXTURES WITH ENHANCED $V_{\ell N}$ IN TEV-SEESAW}

As is well known and also as emphasized in the introduction, for generic forms of both the Dirac mass matrix $m_{D}$ and the $\mathrm{RH}$ neutrino mass matrix $M_{N}$, the seesaw formula in Eq. (2) implies that the heavy-light mixing parameter $V_{\ell N} \simeq \sqrt{m_{\nu} / M_{N}}$ which is a tiny number regardless of whether the seesaw scale is in the $\mathrm{TeV}$ range or higher. This keeps its effect shielded from being probed by either collider or low energy experiments. However, there are some special textures for $m_{D}$ for which even with $\mathrm{TeV}$-scale seesaw, the mixing parameter $V_{\ell N}$ can be significantly enhanced whereas the neutrino masses remain naturally small. We present only one example here to illustrate our case, although several others have been discussed in the literature 10, 11. Consider the matrices $m_{D}$ and $M_{N}$ of the following form:

$$
m_{D}=\left(\begin{array}{lll}
a & \delta_{1} & \epsilon_{1} \\
b & \delta_{2} & \epsilon_{2} \\
c & \delta_{3} & \epsilon_{3}
\end{array}\right) \text { and } M_{N}=\left(\begin{array}{ccc}
0 & M_{1} & 0 \\
M_{1} & \delta M & 0 \\
0 & 0 & M_{2}
\end{array}\right)(3)
$$

with $\epsilon_{i}, \delta_{i} \ll a, b, c$ and $\delta M \ll M_{i}$. In the limit of $\epsilon_{i}, \delta_{i}, \delta M \rightarrow 0$, the neutrino masses vanish, although the heavy-light mixing given by $V_{\ell N_{i}}=m / M_{i}$ (with $m=a, b, c)$ can be quite large. The neutrino masses given by the seesaw formula become proportional to products of $\epsilon_{i}$ and $\delta_{i}$. If by some symmetry one can guarantee the smallness of $\delta_{i}$ and $\epsilon_{i}$, then we have a TeV scale seesaw model with enhanced $V_{\ell N}$. These mass textures can be embedded into L-R models [19] and will have other phenomenological implications, e.g. "large" LFV, violation of unitarity of the PMNS mixing matrix, etc. It is the impact of these scenarios in colliders which is the main focus of the rest of this paper. Note that while we have presented only one example of such non-generic 
Dirac mass matrix in Eq. (3), our following results are also applicable to other Dirac textures discussed in the literature.

\section{THE LEFT-RIGHT PHASE DIAGRAM}

In this section, we present the regions of heavy-light mixing parameter and $\mathrm{RH}$ gauge boson masses where the mixing effects will provide the dominant contribution to the $\ell^{ \pm} \ell^{ \pm} j j$ signal. Clearly there will be flavor dependence in this signal, depending on the underlying Dirac mass texture; we do not discuss those details here and show our results for a generic case. There are four classes of Feynman diagrams in the minimal L-R model which can lead to the $\ell^{ \pm} \ell^{ \pm} j j$ final states (Fig. 1). We denote these diagrams as (a) $L L$, (b) $R R$, (c) $R L$, and (d) $L R$, according to the chirality of the final state lepton-pair. The most widely studied of these are the $L L$ and $R R$ diagrams - the first one in the context of SM-seesaw 8 and the second one in LR models [16, 17. The channel in Fig 1a is a clear probe of the seesaw matrix in both SM-seesaw and L-R seesaw models, but its effectiveness solely relies on the heavy-light mixing $\left|V_{\ell N}\right|^{2}$, and is limited to $M_{N}$ only up to a few hundred GeV. Experimentally, the mass range $M_{N}=100-300 \mathrm{GeV}$ has been explored at the LHC for $\ell=e, \mu$ 20, 21, and direct upper limits on $\left|V_{\ell N}\right|^{2}$ of the order of $10^{-2}-10^{-1}$ have been set. We note here that the complementary limits from electroweak precision tests and lepton flavor violating processes are roughly one to two orders of magnitude stronger (for a review, see Ref. [22]).

In case of Fig 1b, the heavy neutrinos are produced on-shell via the decay of an RH gauge boson and they then subsequently decay into a three-body final state via an off-shell $W_{R}$. This diagram gives the dominant contribution if the heavy-light mixing is assumed to be very small which is of course the naive expectation in the "vanilla" type I seesaw case as noted above. Using this channel, LHC exclusion limits are derived in the $\left(M_{N}, M_{W_{R}}\right)$ plane [23, 24], and currently exclude $M_{W_{R}}$ up to $2.5 \mathrm{TeV}$ for a $\mathrm{TeV}$-scale $M_{N}$. Note that these limits are independent of the Dirac neutrino Yukawa coupling characterizing the heavy-light mixing, and therefore, do not probe the seesaw matrix.

The contributions shown in Figs. 1k and 1 $\mathrm{d}$, on the other hand, necessarily involve the heavy-light neutrino mixing 11 In fact, the $R L$ diagram could give the dominant contribution to the $\ell^{ \pm} \ell^{ \pm} j j$ signal if the mixing $\left|V_{\ell N}\right|$ is non-negligible and/or the $W_{R}$ gauge boson is not too heavy. There are two reasons for this dominance: (i) this contribution leads to a production rate $\sigma\left(p p \rightarrow W_{R} \rightarrow N \ell^{ \pm}\right)$which is independent of mixing and

\footnotetext{
1 The heavy-light mixing also contributes to $0 \nu \beta \beta$ in L-R models 18, 27]. Again these effects are small for generic seesaw matrix, but could be important for large mixing 28].
}
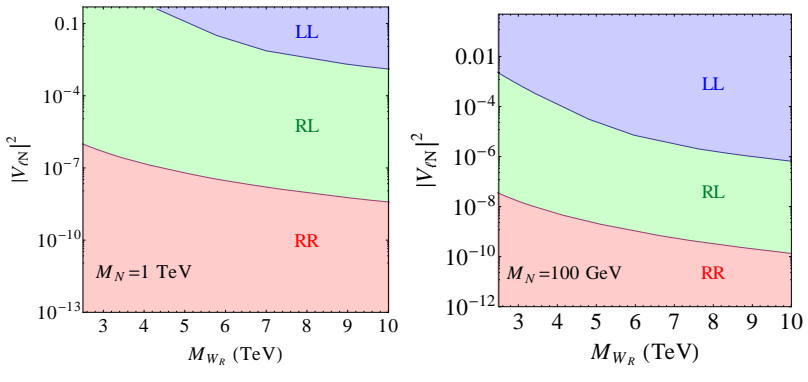

FIG. 2. Phase diagram for the minimal L-R seesaw model.

only suppressed by $\left(M_{W} / M_{W_{R}}\right)^{4}$ (as in the $R R$ case), and can therefore dominate over the $L L$ contribution which depends on $\left|V_{\ell N}\right|^{2}$; (ii) the decay of the heavy neutrino in this case is no longer suppressed by the phase space, since it can have a two-body decay via on-shell $W: N \rightarrow \ell^{ \pm} W \rightarrow \ell^{ \pm} j j$ (as in the $L L$ case). Hence, for a sizable range of the mixing and $\mathrm{RH}$ gauge boson mass, the RL mode is expected to be dominant for the heavy neutrino signal $\ell^{ \pm} \ell^{ \pm} j j$ at the LHC and could constitute a clear probe of the seesaw matrix. It is surprising that this contribution has not been taken into account in the collider analyses so far, although the importance of this contribution has been discussed sporadically, e.g., in the context of a comparative study between heavy Majorana and Dirac neutrinos [25], and in determining the chirality of the heavy gauge boson [26].

The remaining possibility, namely, the $L R$ contribution (Fig. 11) is doubly suppressed by the mixing as well as phase space, and hence, always smaller than at least two of the other three contributions discussed above. Hence, we will not analyze this diagram in details in what follows.

The regions of dominance for various contributions discussed above are shown in Fig. 2 (we call this the "L$\mathrm{R}$ phase diagram") for two typical choices of the heavy neutrino mass $M_{N}=100 \mathrm{GeV}$ and $1 \mathrm{TeV}$. The upper (blue) shaded region with large mixing is where the LL contribution to the $\ell^{ \pm} \ell^{ \pm} j j$ signal is dominant, whereas the lower (red) shaded region with small mixing is dominated by the RR contribution. The middle (green) region is where the RL contribution is dominant and it clearly spans a wide parameter space of the model. In particular, it can probe the seesaw mixing all the way down to $\left|V_{\ell N}\right|^{2} \geq 10^{-8}$, close to the "vanilla" seesaw expectation of $m_{\nu} / M_{N}$.

To further illustrate our point, we compare the magnitudes of signal cross section for the processes shown in Fig. 1 as a function of the RH neutrino mass for a given value of the RH gauge boson mass $M_{W_{R}}$ and the mixing parameter $\left|V_{\ell N}\right|$. This is shown in Fig. 3 for a typical choice of $M_{W_{R}}=3 \mathrm{TeV}$, keeping in mind the current limit from direct collider searches which extend up to $M_{W_{R}}=2.5 \mathrm{TeV}$ [23, 24], and similar lower limits from estimates on the $K_{L}-K_{S}$ mixing 29]. We have only considered $\ell=\mu$ final state for our collider analysis since the 
heavy neutrino mixing to electrons is highly constrained from $0 \nu \beta \beta$ [30]: $M_{W_{R}}^{-4}\left|\sum_{i} V_{e N_{i}}^{2} / M_{N_{i}}\right|<0.1 \mathrm{TeV}^{-5}$. Also we do not consider $\tau$ final states since the $\tau$-lepton identification at the LHC is rather complicated. We have shown the results for $\sqrt{s}=14 \mathrm{TeV}$ LHC and for two sample choices of the mixing: (a) $\left|V_{\ell N}\right|^{2}=3 \times 10^{-3}$, close to the current experimental limit on $\left|V_{\mu N}\right|^{2}$ for a $\mathrm{TeV}$-scale heavy neutrino [31, and (b) the vanilla seesaw expectation: $\left|V_{\ell N}\right|^{2}=\sqrt{\Delta m_{\text {atm }}^{2}} / M_{N}, \Delta m_{\text {atm }}^{2}$ being the atmospheric neutrino mass-squared difference which we take as $2.35 \times 10^{-3} \mathrm{eV}^{2}$ 32]

The heavy neutrino signal cross section is given by

$$
\begin{aligned}
\sigma\left(p p \rightarrow N \ell^{ \pm} \rightarrow \ell^{ \pm} \ell^{ \pm} j j\right)= & \sigma_{\operatorname{prod}}\left(p p \rightarrow W_{L, R} \rightarrow N \ell^{ \pm}\right) \\
& \times \operatorname{BR}\left(N \rightarrow \ell^{ \pm} j j\right) .
\end{aligned}
$$

The parton-level production cross sections were generated for $\sqrt{s}=14 \mathrm{TeV}$ using CalcHEP 33 , with the CTEQ6L parton distribution functions 34]. For the $L L$ and $R L$ modes, we have the 2-body decay $N \rightarrow \ell^{ \pm} W$ followed by $W \rightarrow j j$, with the corresponding branching ratio

$$
\mathrm{BR}\left(N \rightarrow \ell^{ \pm} j j\right)=\frac{\Gamma\left(N \rightarrow \ell^{ \pm} W\right)}{\Gamma_{N}^{\text {tot }}} \times \mathrm{BR}(W \rightarrow j j),
$$

where $\operatorname{BR}(W \rightarrow j j)=0.676$ 32. For the $R R$ mode, we have the three-body decay $N \rightarrow \ell^{ \pm} W_{R}^{*} \rightarrow \ell^{ \pm} j j$. The total decay width $\Gamma_{N}^{\text {tot }}$ is the sum of partial widths to 2-body final states (when kinematically allowed):

$$
\begin{gathered}
\Gamma\left(N \rightarrow \ell^{ \pm} W\right)=\frac{g^{2}\left|V_{\ell N}\right|^{2}}{64 \pi} \frac{M_{N}^{3}}{M_{W}^{2}}\left(1-\frac{M_{W}^{2}}{M_{N}^{2}}\right)^{2}\left(1+2 \frac{M_{W}^{2}}{M_{N}^{2}}\right), \\
\Gamma\left(N \rightarrow \nu_{\ell} Z, \bar{\nu}_{\ell} Z\right)=\frac{g^{2}\left|V_{\ell N}\right|^{2}}{128 \pi \cos ^{2} \theta_{W}} \frac{M_{N}^{3}}{M_{Z}^{2}}\left(1-\frac{M_{Z}^{2}}{M_{N}^{2}}\right)^{2} \\
\times\left(1+2 \frac{M_{Z}^{2}}{M_{N}^{2}}\right),
\end{gathered}
$$$$
\Gamma\left(N \rightarrow \nu_{\ell} h, \bar{\nu}_{\ell} h\right)=\frac{g^{2}\left|V_{\ell N}\right|^{2}}{128 \pi} \frac{M_{N}^{3}}{M_{W}^{2}}\left(1-\frac{M_{h}^{2}}{M_{N}^{2}}\right)^{2},
$$

and 3-body final states (in the limit of massless final states, and assuming $W_{R}, Z_{R}$ highly off-shell):

$$
\begin{aligned}
& \Gamma\left(N \rightarrow \ell^{ \pm} W_{R}^{*} \rightarrow \ell^{ \pm} j j\right) \simeq \frac{3 g_{R}^{4}}{2048 \pi^{3}} \frac{M_{N}^{5}}{M_{W_{R}}^{4}}, \\
& \Gamma\left(N \rightarrow \nu_{\ell}\left(\bar{\nu}_{\ell}\right) Z_{R}^{*} \rightarrow \nu_{\ell}\left(\bar{\nu}_{\ell}\right) j j\right) \simeq \frac{3 g_{R}^{4}}{4096 \pi^{3}} \frac{\cos ^{8} \theta_{W}}{\cos ^{2} 2 \theta_{W}} \frac{M_{N}^{5}}{M_{Z_{R}}^{4}} .
\end{aligned}
$$

For numerical purposes, we use $m_{h}=125 \mathrm{GeV}$, $g_{L}=g_{R}$ for the weak gauge couplings, and the relation $M_{Z_{R}} / M_{W_{R}}=\cos \theta_{W} / \sqrt{\cos 2 \theta_{W}}$ (where $\theta_{W}$ is the Weinberg angle), assuming that the LR-symmetry is broken by an $S U(2)_{R}$ triplet Higgs vacuum expectation value. We neglect the contribution of the 3-body decay modes of $N$ mediated by the $S U(2)_{L}$ triplet Higgs fields to its total width, since it not only involves the heavy-light mixing $\left|V_{\ell N}\right|^{2}$ (as the $N \rightarrow \ell W$ mode) but is further suppressed by the factor $M_{N}^{5} / M_{\Delta_{L}}^{4}$ (assuming $M_{\Delta_{L}} \gg M_{N}$ ) as well as the 3 -body phase space.
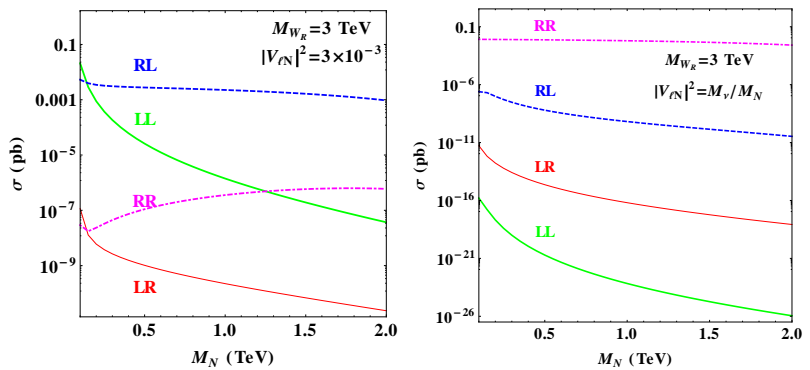

FIG. 3. Comparison of the signal cross sections for various modes shown in Fig. 1 for two benchmark scenarios.

It is clear from Fig. 3 that for small heavy-light neutrino mixing (right panel), the $R R$ mode is dominant for a TeV-scale $W_{R}$, while for large mixing (left panel), the $R L$ mode is dominant over both $L L$ and $R R$ modes over a wide range of $\mathrm{RH}$ neutrino masses relevant for their collider searches. Hence for consistency the $R L$ mode must also be taken into account in the collider analysis of heavy neutrinos in a TeV-scale LR-model.

\section{IMPROVED COLLIDER LIMITS ON THE HEAVY-LIGHT NEUTRINO MIXING}

As an immediate implication of our results shown above, we can derive improved collider limits on the left-right neutrino mixing compared to the existing limits 20, 21] obtained from $\sqrt{s}=7 \mathrm{TeV}$ LHC data assuming the inclusive signal cross section for the $L L$ mode alone. For the range of mixing parameter being constrained here, the $R L$ contribution is in general dominant, especially for higher $M_{N}$ (cf. Fig. 3) and the total $(L L+R L)$ inclusive cross section is larger thus yielding a stronger limit on the mixing parameter. We do not include the $R R$ contribution to the signal cross section since it is sub-dominant for the range of $\left|V_{\ell N}\right|$ considered here, and moreover, this channel will have a significantly smaller efficiency after applying the selection cuts designed for $L L$ mode [20, 21] (also valid for $R L$ mode)in particular, the requirement of the dijet invariant mass $m_{j j}$ close to $M_{W}$.

Thus, given an experimentally observed limit on the signal cross section $\sigma_{\text {expt }}$, we can infer the following: (i) the $\left(M_{N}, M_{W_{R}}\right)$ plane for which $\sigma_{R L} \geq \sigma_{\text {expt }}$ is ruled out, thus providing a complementary probe of this parameter space which is currently probed at the LHC only in the $R R$ mode [23, 24]; (ii) for $\sigma_{R L}<\tilde{\sigma}_{L L}<\sigma_{\text {expt }}$ where $\tilde{\sigma}_{L L} \equiv \sigma_{L L} /\left|V_{\ell N}\right|^{2}$ is the normalized $L L$ cross section, an improved limit on the mixing parameter can be derived:

$$
\left|V_{\ell N}\right|^{2}<\frac{\sigma_{\operatorname{expt}}-\sigma_{R L}}{\tilde{\sigma}_{L L}}
$$

which is obviously stronger than that derived assuming $\sigma_{R L}=0$. Using the observed cross section limit for $\sqrt{s}=7 \mathrm{TeV}$ from the ATLAS analysis [21, we find the improvement in the upper limit on $\left|V_{\ell N}\right|^{2}$ taking into 
account the combined $(L L+R L)$ mode in the minimal LR model with $M_{W_{R}}=2.5 \mathrm{TeV}$ to be about $10 \%$ for $M_{N}=300 \mathrm{GeV}$, and somewhat lower for decreasing (increasing) $M_{N}\left(M_{W_{R}}\right)$. However, we expect it to be much more prominent for higher values of $M_{N}$ and/or at $\sqrt{s}=14 \mathrm{TeV}$ LHC due to the enhanced $R L$ cross section as shown in Fig. 3. For illustration, assuming the expected upper limit on the signal cross section at $\sqrt{s}=14 \mathrm{TeV}$ LHC to be smaller than the observed limit at $\sqrt{s}=7 \mathrm{TeV}$, we obtain conservative upper limits on the mixing parameter as shown in Table/I. The low $M_{W_{R}}$ points denoted by a ${ }^{*}$ predict cross sections larger than our assumed experimental limit, and hence, can be ruled out in case of no positive signal. On the other hand, for the allowed region, the improvement in the limit on mixing could be as large as $60 \%$.

\begin{tabular}{c|c||c|c|c}
\hline \hline Mode & \multicolumn{2}{|c||}{$\begin{array}{c}M_{W_{R}} \\
\end{array}$} & \multicolumn{2}{|c}{ Upper limit on $\left|V_{\ell N}\right|^{2}$ for $\sqrt{s}=14 \mathrm{TeV}$ LHC } \\
\cline { 2 - 5 } & $M_{N}=100 \mathrm{GeV}$ & $M_{N}=200 \mathrm{GeV}$ & $M_{N}=300 \mathrm{GeV}$ \\
\hline \multirow{3}{*}{$L L$} & 2.5 & $*$ & $*$ & $*$ \\
\cline { 2 - 5 }+ & 3 & 0.0005 & $*$ & $*$ \\
\cline { 2 - 5 }$R L$ & 3.5 & 0.0009 & $*$ & $*$ \\
\cline { 2 - 5 } & 4 & 0.0011 & 0.0013 & 0.0042 \\
\cline { 2 - 5 } & 5 & 0.0012 & 0.0026 & 0.0092 \\
\hline \multicolumn{2}{c||}{$L L$} & 0.0012 & 0.0029 & 0.0102 \\
\hline \hline
\end{tabular}

TABLE I. Projected upper limits on the heavy-light neutrino mixing in the minimal LR model for $\sqrt{s}=14 \mathrm{TeV}$ LHC. The * points predict a cross section larger than our expected $\sigma_{\text {expt }}$.

\section{POST-DISCOVERY DISTINCTION}

Here we propose a possible distinction between the various contributions shown in Fig. 2 by considering two kinematic variables, namely, the dilepton invariant mass distribution and angular correlation between the charged leptons. For a realistic collider simulation, the parton-level signal events generated by CalcHEP [33] are fed into PYTHIA 35] and PGS4 36] to implement parton showering, hadronization and detector effects. We have used an anti- $k_{T}$ jet algorithm with jet cone size parameter $R=0.4$. Apart from the basic selection criteria of two same-sign muons and two light jets, we have implemented the following selection cuts for both $L L$ and $R L$ modes following the latest ATLAS analysis [21]: $p_{T}^{j}>20 \mathrm{GeV}, p_{T}^{\ell}>20 \mathrm{GeV}, p_{T}^{\ell, \text { leading }}>25$ $\mathrm{GeV},|\eta(j)|<2.8,|\eta(\ell)|<2.5, \mathbb{E}_{T}<35 \mathrm{GeV}$ and $m_{j j} \in[55,120] \mathrm{GeV}$. For the $R R$ mode, we have implemented the cuts following the latest CMS analysis [24]: $M_{\ell \ell j j}>600 \mathrm{GeV}, M_{\ell \ell}>200 \mathrm{GeV}, p_{T}^{j}>40 \mathrm{GeV}$, $p_{T}^{\ell}>40 \mathrm{GeV}, p_{T}^{\ell, \text { leading }}>60 \mathrm{GeV},|\eta(j)|<3.0$ and $|\eta(\ell)|<2.5$. For comparison, all distributions have been normalized to unity after applying the cuts. The simulation results for an illustrative case with $\left|V_{\ell N}\right|^{2}=0.003$,
$M_{W_{R}}=3 \mathrm{TeV}$ and $M_{N}=1 \mathrm{TeV}$ are shown in Fig. 4. It is clear that the dilepton invariant mass distribution
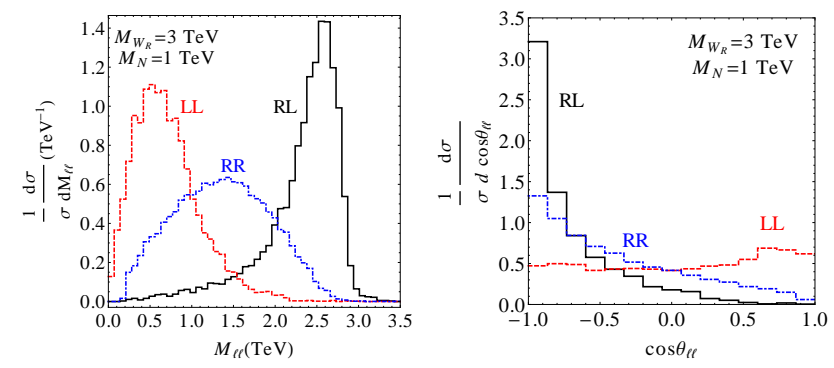

FIG. 4. The invariant mass distribution and the angular correlation of the final state leptons for the $L L, R L$ and $R R$ modes shown in Fig. 1. For comparison, all distributions have been normalized to unity.

(left panel) is a good kinematic variable for distinction between the $L L, R L$ and $R R$ modes at the LHC. The angular correlation between the two leptons (right panel) is another good variable to distinguish the $R L$ and $R R$ case from the $L L$ case due to different helicity correlations.

\section{CONCLUSION}

In summary, we have pointed out a new contribution to the smoking gun collider signals of a $\mathrm{TeV}$ scale leftright seesaw model i.e. $\ell^{ \pm} \ell^{ \pm} j j$ coming from the heavylight neutrino mixing contribution (called $R L$ in the text) which can dominate over the usually discussed $L L$ and $R R$ contributions. Probing this contribution can provide crucial information on the detailed nature of the seesaw mechanism and supplement searches for this effect using violations of unitarity of the PMNS matrix. This will provide extremely important information regarding the detailed nature of left-right $\mathrm{TeV}$ scale seesaw models. We emphasize the importance of this channel for heavy Majorana neutrino searches in the hope that this will be taken into account in the future experimental analyses, along with the usual $L L$ and $R R$ channels. We show how taking into account this $R L$ contribution can improve the collider limits on the left-right neutrino mixing in certain parameter domains of the seesaw matrix, with the improvement becoming more prominent as we go to higher heavy neutrino masses and higher center of mass energy at the LHC. We also propose a simple way to distinguish the different contributions and to identify the dominant channel by analyzing the invariant mass distribution and angular correlation of the two same-sign leptons. Should a same-sign dilepton plus two jets with no missing energy signal be observed at the LHC, this will help us in determining the existence of a TeV-scale LR-symmetry as well as the structure of the seesaw matrix.

Acknowledgments: C-Y.C and P.S.B.D. thank Ian Lewis and Apostolos Pilaftsis for helpful discussions. P.S.B.D. also thanks the High Energy Theory group at 
BNL for hospitality where part of this work was carried out. The work of C-Y.C. is supported by the US Department of Energy under Grant DE-AC02-98CH10886, P.S.B.D. is supported by the Lancaster-Manchester-
Sheffield Consortium for Fundamental Physics under STFC grant ST/J000418/1, and R.N.M. is supported by National Science Foundation grant No. PHY-0968854.
[1] P. Minkowski, Phys. Lett. B 67, 421 (1977); T. Yanagida, Workshop on unified theories and baryon number in the universe, eds. A. Sawada and A. Sugamoto, KEK, Tsukuba (1979); M. Gell-Mann, P. Ramond and R. Slansky, Supergravity, ed. P. Van Niewenhuizen and D. Freeman, North Holland, Amsterdam (1980); R. N. Mohapatra and G. Senjanović, Phys. Rev. Lett. 44, 912 (1980).

[2] J. Schechter and J. W. F. Valle, Phys. Rev. D 22, 2227 (1980); Phys. Rev. D 25, 774 (1982).

[3] For reviews, see e.g., W. Rodejohann, Int. J. Mod. Phys. E 20, 1833 (2011); J. D. Vergados, H. Ejiri and F. Simkovic, Rept. Prog. Phys. 75, 106301 (2012).

[4] S. Antusch, C. Biggio, E. Fernandez-Martinez, M. B. Gavela and J. Lopez-Pavon, JHEP 0610, 084 (2006); A. Abada, C. Biggio, F. Bonnet, M. B. Gavela and T. Hambye, JHEP 0712, 061 (2007); M. Malinsky, T. Ohlsson and H. Zhang, Phys. Rev. D 79, 073009 (2009); M. Malinsky, T. Ohlsson, Z. -z. Xing and H. Zhang, Phys. Lett. B 679, 242 (2009); P. S. B. Dev and R. N. Mohapatra, Phys. Rev. D 81, 013001 (2010).

[5] For a review, see e.g., M. Drewes, arXiv:1303.6912 [hep$\mathrm{ph}]$.

[6] J.C. Pati and A. Salam, Phys. Rev. D 10, 275 (1974); R. N. Mohapatra and J. C. Pati, Phys. Rev. D 112558 (1975); R. N. Mohapatra and G. Senjanović, Phys. Rev. D 121502 (1975).

[7] G. Senjanović, Int. J. Mod. Phys. A 26, 1469 (2011).

[8] A. Datta, M. Guchait and A. Pilaftsis, Phys. Rev. D 50, 3195 (1994); F. M. L. Almeida, Jr. et al., Phys. Rev. D 62, 075004 (2000); T. Han and B. Zhang, Phys. Rev. Lett. 97, 171804 (2006); F. del Aguila, J. A. AguilarSaavedra and R. Pittau, JHEP 10, 047 (2009); F. del Aguila and J. A. Aguilar-Saavedra, Nucl. Phys. B $\mathbf{8 1 3}$, 22 (2009).

[9] P. A. R. Ade et al. [Planck Collaboration], arXiv:1303.5076 [astro-ph.CO].

[10] A. Pilaftsis, Z. Phys. C 55, 275 (1992); J. Gluza, Acta Phys. Polon. B 33, 1735 (2002); J. Kersten and A. Y. Smirnov, Phys. Rev. D 76, 073005 (2007); A. de Gouvea, arXiv:0706.1732 [hep-ph]; Z. -z. Xing, Prog. Theor. Phys. Suppl. 180, 112 (2009); X. -G. He, S. Oh, J. Tandean and C. -C. Wen, Phys. Rev. D 80, 073012 (2009); A. Ibarra, E. Molinaro and S. T. Petcov, JHEP 1009, 108 (2010); N. Haba, T. Horita, K. Kaneta and Y. Mimura, arXiv:1110.2252 [hep-ph].

[11] M. Mitra, G. Senjanovic and F. Vissani, Nucl. Phys. B 856, 26 (2012).

[12] M. Blennow, E. Fernandez-Martinez, J. Lopez-Pavon and J. Menendez, JHEP 1007, 096 (2010).

[13] A. Ibarra, E. Molinaro and S. T. Petcov, Phys. Rev. D 84, 013005 (2011); J. Lopez-Pavon, S. Pascoli and C. f. Wong, arXiv:1209.5342 [hep-ph].

[14] V. Tello, M. Nemevsek, F. Nesti, G. Senjanović and F. Vissani, Phys. Rev. Lett. 106, 151801 (2011).

[15] R. N. Mohapatra and G. Senjanović, in Ref. 1]; Phys.
Rev. D 23, 165 (1981).

[16] W.-Y. Keung and G. Senjanović, Phys. Rev. Lett. 50, 1427 (1983).

[17] A. Datta, M. Guchait and D. P. Roy, Phys. Rev. D 47, 961 (1993); A. Ferrari et al., Phys. Rev. D 62, 013001 (2000); S. N. Gninenko, M. M. Kirsanov, N. V. Krasnikov and V. A. Matveev, Phys. Atom. Nucl. 70, 441 (2007); M. Nemevsek, F. Nesti, G. Senjanović and Y. Zhang, Phys. Rev. D 83, 115014 (2011); J. Chakrabortty, J. Gluza, R. Sevillano and R. Szafron, JHEP 1207, 038 (2012); S. P. Das, F. F. Deppisch, O. Kittel and J. W. F. Valle, Phys. Rev. D 86, 055006 (2012); J. A. Aguilar-Saavedra and F. R. Joaquim, Phys. Rev. D 86, 073005 (2012).

[18] R. N. Mohapatra, Phys. Rev. D 34, 909 (1986).

[19] P. S. B. Dev, C. Lee and R. N. Mohapatra, work in progress.

[20] CMS Collaboration, Phys. Lett. B 717, 109 (2012).

[21] ATLAS Collaboration, ATLAS-CONF-2012-139.

[22] A. Atre, T. Han, S. Pascoli and B. Zhang, JHEP 0905, 030 (2009).

[23] ATLAS Collaboration, Eur. Phys. J. C 72, 2056 (2012).

[24] CMS Collaboration, CMS-PAS-EXO-12-017.

[25] C. -Y. Chen and P. S. B. Dev, Phys. Rev. D 85, 093018 (2012).

[26] T. Han, I. Lewis, R. Ruiz and Z. -g. Si, Phys. Rev. D 87, 035011 (2013).

[27] M. Hirsch, H. V. Klapdor-Kleingrothaus and O. Panella, Phys. Lett. B 374, 7 (1996).

[28] M. Nemevsek, G. Senjanović and V. Tello, Phys. Rev. Lett. 110, 151802 (2013); J. Barry and W. Rodejohann, arXiv:1303.6324 [hep-ph].

[29] G. Beall, M. Bander and A. Soni, Phys. Rev. Lett. 48, 848 (1982); H. An, X. Ji, R. N. Mohapatra, and Y. Zhang, Nucl. Phys. B 802, 247 (2008); A. Maiezza, M. Nemevsek, F. Nesti and G. Senjanović, Phys. Rev. D 82, 055022 (2010).

[30] P. Benes, A. Faessler, F. Simkovic and S. Kovalenko, Phys. Rev. D 71, 077901 (2005); P. S. B. Dev, S. Goswami, M. Mitra and W. Rodejohann, arXiv:1305.0056 [hep-ph].

[31] F. del Aguila, J. de Blas and M. Perez-Victoria, Phys. Rev. D 78, 013010 (2008).

[32] J. Beringer et al. (Particle Data Group), Phys. Rev. D 86, 010001 (2012).

[33] A. Pukhov et al., hep-ph/9908288 A. Pukhov, hep$\mathrm{ph} / 0412191$.

[34] J. Pumplin et al., JHEP 07, 012 (2002).

[35] T. Sjostrand, S. Mrenna, and P. Z. Skands, JHEP 05, 026 (2006).

[36] J. Conway, http://www.physics.ucdavis.edu/ conway/

research/software/pgs/pgs4-general.htm. 